\title{
Ablation d'un film d'or par laser à excimère
}

\author{
M. Sentis, J. Hermann, A. Pereira, Ph. Delaporte, W. Marine ${ }^{1}$, J. Perrière ${ }^{2}$, \\ L. Bianchi ${ }^{3}$ et R. Galli ${ }^{4}$
}

\author{
LP3, FRE 2165 du CNRS, Faculté des Sciences de Luminy, Case 917, \\ 13288 Marseille cedex 9 , France \\ ${ }^{1}$ Groupe de Physique des États Condensés (GPEC), UMR 6631 du CNRS, \\ Université de la Méditerranée, France \\ ${ }^{2}$ Groupe de Physique des Solides, UMR 7588 du CNRS, Université Paris VI, France \\ ${ }^{3}$ Commissariat à l'Énergie Atomique/LR, BP. 16, 37260 Monts, France \\ ${ }^{4}$ Commissariat à l'Énergie Atomique/DIF, BP. 12, 91680 Bruyères-le-Châtel, France
}

\begin{abstract}
Résumé : Ces travaux de recherche ont été réalisés dans le cadre du projet de développement du Laser MégaJoule (LMJ). Parmi les expériences d'interaction laser - matière à très haut flux, certaines conduiront à un dépôt de particules d'or sur les éléments internes de la chambre d'interaction. Pour nettoyer ce dépôt de particules, la possibilité d'utiliser un procédé automatisé basé sur l'ablation laser a été étudiée. Un modèle numérique simplifié a été développé et une étude expérimentale réalisée sur des échantillons d'acier inoxydable ou de $\mathrm{B}_{4} \mathrm{C}$ recouverts d'un film d'or d'une épaisseur de $\sim 20 \mathrm{~nm}$ déposés par PVD. Ces travaux montrent que plus de $95 \%$ du film d'or peuvent être enlevés avec quelques tirs d'un laser XeCl dès que la densité d'énergie dépasse $3 \mathrm{~J} / \mathrm{cm}^{2}$.
\end{abstract}

\section{INTRODUCTION}

Le nettoyage de surfaces par lasers impulsionnels est un procédé qui offre de multiples avantages tels que procédé sans contact et automatisable, réduction des déchets primaires, absence de déchets secondaires, etc. Tous ces avantages encouragent le développement de nouvelles recherches dédiées à des applications spécifiques de l'ablation laser au nettoyage. C'est dans cette mouvance que l'étude présentée ici a été conduite. Il s'agissait de déterminer les paramètres lasers (longueur d'onde, durée d'impulsion, densité d'énergie, taux de recouvrement) permettant de nettoyer efficacement une couche d'or d'environ $20 \mathrm{~nm}$ recouvrant un substrat en acier inoxydable ou en carbure de bore $\left(\mathrm{B}_{4} \mathrm{C}\right)$. Cette recherche a été réalisée dans le cadre du projet de développement du Laser MégaJoule. En effet, les expériences d'interaction qui seront menées avec cette source laser de très forte puissance provoqueront un dépôt de particules métalliques (principalement de l'or) sur les parois intérieures de la chambre d'interaction. Ce dépôt devra être éliminé périodiquement pour éviter notamment une pollution des fenêtres optiques. La possibilité d'utiliser un procédé sec de nettoyage par laser UV a donc été étudié.

\section{ETUDE NUMERIQUE 1D}

Cette étude simplifiée a été effectuée en utilisant un modèle ID thermique tenant compte des caractéristiques du laser et des caractéristiques optiques et thermiques des matériaux. Le but de cette étude est d'étudier l'influence de la longueur d'onde et de la durée de l'impulsion laser sur le seuil d'ablation et la profondeur du bain de fusion. Elle est basée sur la résolution de l'équation de la chaleur unidimensionnelle suivante :

$$
\rho(T) C(T) \frac{\partial T(x, t)}{\partial t}=\frac{\partial}{\partial x}\left[k_{t h}(T) \frac{\partial T(x, t)}{\partial x}\right]+G(x, t)
$$

où $k_{t h}$ est la conductivité thermique, $\rho$ la densité spécifique, $C$ la capacité calorifique du matériau, $T$ la température, $x$ la profondeur dans le matériau et $G$ la source de chaleur due à l'absorption du faiscean laser. 
L'équation de la chaleur unidimensionnelle peut être intégrée numériquement en utilisant la méthode des différences finies. Le matériau est alors considéré comme un ensemble de $n$ couches d'épaisseur $\Delta x$ placées à la profondeur $x_{m}=m \Delta x$ dont la température évolue dans le temps divisé en intervalles $\Delta t$ avec $t_{p}=p \Delta t$. Les conditions limites sont les suivantes:

$$
\begin{aligned}
& T(t=0)=T_{a m b} \\
& T(x \rightarrow \infty)=T_{a m b} \\
& \left.\frac{\partial T(x, t)}{\partial x}\right|_{x=0}=0
\end{aligned}
$$

La condition (a) signifie que la température initiale est égale à la température ambiante $T_{a m b}=300 \mathrm{~K}$ sur toute la profondeur. Le matériau étant considéré comme semi-infini, la température de la couche la plus profonde reste constante à $T_{a m b}$ pendant toute la durée de l'interaction laser-matière (b). En négligeant les pertes de chaleur par radiation et par convection, le flux de chaleur à travers la surface est nul (c). Cette dernière condition n'est satisfaite qu'en absence de vaporisation. Cependant, une description approximative du régime d'ablation est possible en introduisant la chaleur latente de vaporisation comme un terme de perte de chaleur.

Le modèle tient compte : - de la variation des propriétés du matériau en fonction de la température

- des enthalpies de fusion et de vaporisation $\Delta H_{\text {fus }}$ et $\Delta H_{\text {vap }}$.

La température de vaporisation $T_{v a p}$ est obtenue à l'aide de l'équation de Clausius-Clapeyron en supposant que la pression de vapeur soit égale à la pression atmosphérique (large approximation : l'ablation laser est un processus hors équilibre thermodynamique, et la pression de vapeur augmente considérablement). Toutefois, comme $\Delta H_{v a p}$ est très grand par rapport à l'énergie nécessaire pour chauffer la matière jusqu'au point de vaporisation, l'incertitude sur $T_{\text {vap }}$ a une faible influence sur le calcul de la quantité de matière ablatée.

A titre d'exemple, l'évolution temporelle de la température et en profondeur est présentée figure 1 pour un échantillon d'or semi-infini (a) et pour une couche mince d'or de $20 \mathrm{~nm}$ sur un substrat de conductivité thermique de $0.3 \mathrm{~W} \mathrm{~cm}^{-1} \mathrm{~K}^{-1}$ correspondant au $\mathrm{B}_{4} \mathrm{C}$ ou à l'acier inoxydable (b). Les échantillons ont été irradiés à l'aide d'un laser $\mathrm{XeCl}$ d'une durée d'ipmulsion $\tau_{\text {las }}=15 \mathrm{~ns}$ et une fluence légèrement supérieure à celle du seuil d'ablation.

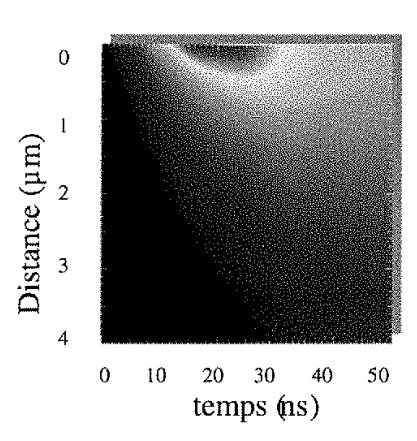

(a)

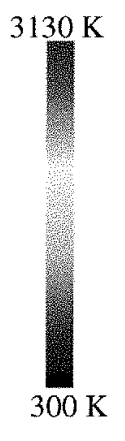

$300 \mathrm{~K}$

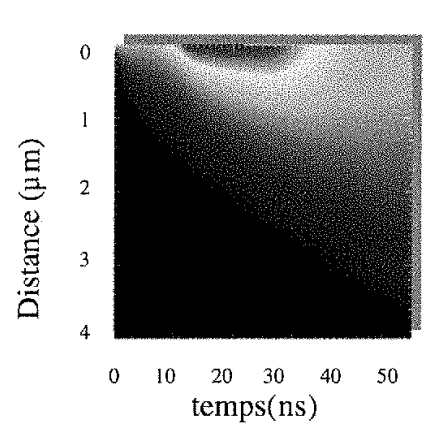

(b)

Figure 1: Evolution de la température pour de l'or (a) et une couche d'or sur inox (b) dans le cas d'une impulsion laser XeCl avec $\tau=15$ ns et une fluence au-dessus du seuil d'ablation.

A l'instant $t=0$ l'échantillon est à la température ambiante représentée par la couleur noire sur toute la profondeur suivant la condition limite (2a). Par la suite la température augmente rapidement au voisinage de 
la surface due au chauffage par laser. En profondeur, la température augmente plus lentement par diffusion thermique. Par rapport à l'or semi-infini (fig. 1a), l'augmentation de la température de surface est plus rapide pour la couche mince qui atteint la température de fusion $T_{f u s}$ en moins d'une nanoseconde (Fig. 1b). Cette augmentation rapide est une conséquence du faible coefficient de conductivité thermique du substrat.

La figure 2 montre la profondeur de la couche d'or ablatée par irradiation laser $\mathrm{XeCl}$ en fonction de la fluence pour trois durées d'impulsion. L'influence de la longueur d'onde du laser sur le processus d'ablation de la couche d'or est montrée à l'aide de la figure 3 où est donnée l'évolution de l'épaisseur ablatée en fonction de la fluence pour des impulsions d'une durée $\tau_{\text {las }}=15 \mathrm{~ns}$. Les valeurs du seuil d'ablation sont affichées dans le tableau I pour trois durées d'impulsion et trois types de laser (Nd :YAG, XeCl, ArF). On note, que par rapport à l'or semi-infini (tableau II) le seuil d'ablation de la couche d'or est presque d'un ordre de grandeur inférieur.

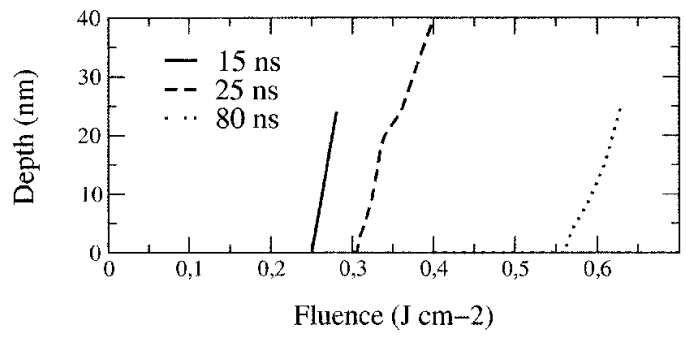

Figure 2: Epaisseur ablatée en fonction de la fluence pour la couche d'or irradiée par laser XeCl.

\begin{tabular}{cccc}
\hline Laser & $15 \mathrm{~ns}$ & $25 \mathrm{~ns}$ & $80 \mathrm{~ns}$ \\
\hline $\mathrm{XeCl}$ & 0.26 & 0.31 & 0.57 \\
$\mathrm{ArF}$ & 0.26 & & \\
$\mathrm{Nd}: \mathrm{YAG}$ & 3.2 & - & - \\
\hline
\end{tabular}

Tableau I: Fluence seuil $F_{\text {las }}$ en $\mathrm{Jcm}^{-2}$ pour l'ablation de la couche d'or.

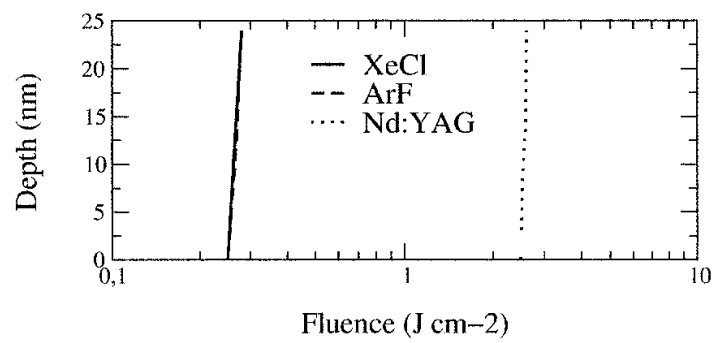

Figure 3: Epaisseur ablatée en fonction de la fluence pour la couche d'or $\left(\tau_{\text {tas }}=15 \mathrm{~ns}\right.$.)

\begin{tabular}{cccc} 
Laser & $15 \mathrm{~ns}$ & $25 \mathrm{~ns}$ & $80 \mathrm{~ns}$ \\
\hline $\mathrm{XeCl}$ & $1.6 / 0.80$ & $2.2 / 1.06$ & $4.6 / 2.0$ \\
$\mathrm{ArF}$ & $1.4 / 0.82$ & $1.8 / 1.06$ & $3.8 / 1.84$ \\
$\mathrm{Nd} \mathrm{YAG}$ & $>6$ & $>6$ & $>6$ \\
\hline
\end{tabular}

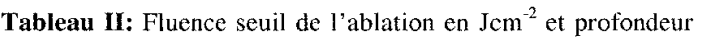
du bain de fusion correspondante en $\mu \mathrm{m} F_{l a s} / x_{f i t s}$ pour l'or semiinfini.

\section{ETUDE EXPERIMENTALE}

Les éprouvettes $\left(1 \times 1 \mathrm{~cm}^{2}\right)$ ont été irradiées à l'aide d'un laser $\mathrm{XeCl}(\lambda=308 \mathrm{~nm})$ ayant les caractéristiques suivantes : une durée d'impulsion à mi-hauteur $\tau=25 \mathrm{~ns}$, une énergie par impulsion de l'ordre de $350 \mathrm{~mJ}$ et un taux de répétition maximum $200 \mathrm{~Hz}$. Elles ont été fixées sur une table XY motorisée dont les déplacements sont synchronisés par rapport au déclenchement du Jaser. Une buse d'aspiration permet la récupération des particules éjectées lors du traitement laser. Un masque placé en sortie du laser sert à sélectionner la partie la plus homogène du faisceau laser. Tous les échantillons ont été traités à l'air sur toute leur surface avec un recouvrement entre chaque tir de $50 \%$ dans les deux directions. Les échantillons ont ensuite été analysés par différents moyens : XPS, MEB, Auger, et RBS.

L'évolution du pourcentage atomique d'or résiduel, analysé par XPS, en fonction de la densité d'énergie laser est montrée sur la figure 4 pour l'acier inoxydable et sur la figure 5 pour le $\mathrm{B}_{4} \mathrm{C}$. L'épaisseur analysée est de $10 \mathrm{~nm}$. On constate que le seuil d'ablation se situe en dessous de $1 \mathrm{~J} / \mathrm{cm}^{2}$ et que le pourcentage d'or diminue très rapidement pour être de l'ordre de $0,4 \%$ à $5 \mathrm{~J} / \mathrm{cm}^{2}$ et 8 tirs pour l'acier inoxydable et de $2 \%$ pour le $\mathrm{B}_{4} \mathrm{C}$ dans les même conditions d'irradiation. 


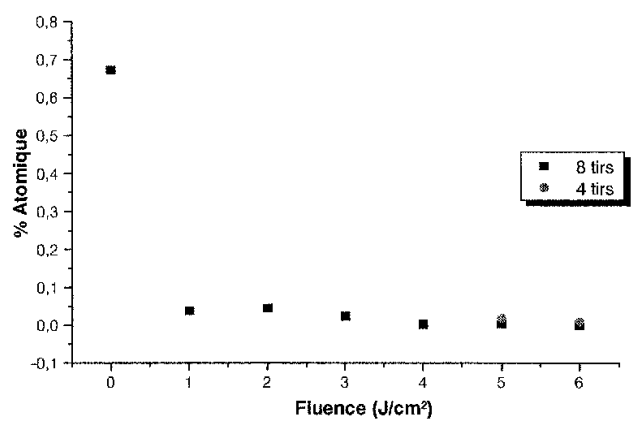

Figure 4 : Evolution du \% atomique d'or pour un substrat en acier inoxydable après irradiation laser

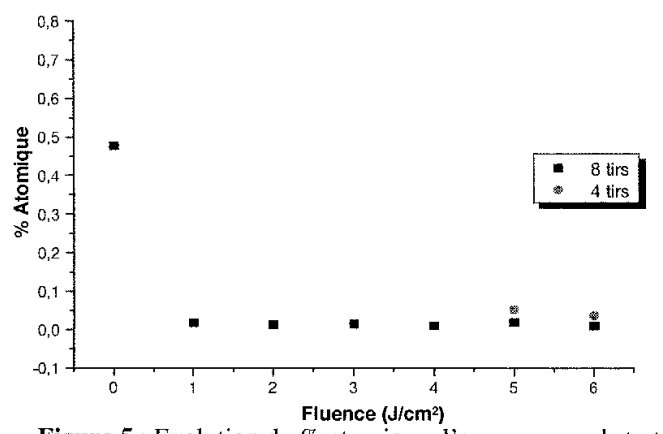

Figure 5 : Evolution du $\%$ atomique d'or pour un substrat en $\mathrm{B}_{4} \mathrm{C}$ après irradiation laser

Les observations par MEB montrent un effet de "lissage" de la surface. A partir de $4 \mathrm{~J} / \mathrm{cm}^{2}$, on observe la présence de nanostructures, absentes à $2 \mathrm{~J} / \mathrm{cm}^{2}$. Une analyse Auger de la composition du matériau sur une profondeur de $1 \mu \mathrm{m}$ a été effectuée. Le pourcentage atomique d'or après irradiation est de $7 \%$ et $3 \%$ pour des densités d'énergie respectives de 2 et $4 \mathrm{~J} / \mathrm{cm}^{2}$ et des substrats en acier inoxydable.

En comparaison avec les résultats d'analyses XPS $\left(4,5 \%\right.$ et $0,3 \%$ à 2 et $\left.4 \mathrm{~J} / \mathrm{cm}^{2}\right)$, ces chiffres sont plus élevés. Deux raisons peuvent expliquer cette différence : la profondeur du matériau analysée et la méthode d'analyse. En effet par méthode XPS la profondeur analysée n'est que de $10 \mathrm{~nm}$ alors que par Auger, elle est de $1 \mu \mathrm{m}$. Lors de l'ablation de l'or peut diffuser sur une profondeur plus importante que $10 \mathrm{~nm}$. Par ailleurs, par analyse Auger il est impossible de prendre en compte des éléments légers tels que l'oxygène et le carbone. A faible densité d'énergie, $2 \mathrm{~J} / \mathrm{cm}^{2}$, les résultats d'analyses XPS et Auger sont du même ordre de grandeur $(4,5 \%$ et $7 \%$ respectivement), à cette densité d'énergie la formation d'oxydes et de carbures est moindre. Par contre à $4 \mathrm{~J} / \mathrm{cm}^{2}$, la formation d'oxydes et de carbures devient très importante, la mesure par Auger ne tenant pas compte de la présence d'oxygène et de carbone, surestime donc la proportion d'or par rapport à l'ensemble des composants ce qui peut expliquer la différence importante entre les mesures $(0,3 \%$ pour XPS et 3\% pour Auger). L'analyse Auger a été effectuée dans toutes les différentes zones d'irradiation et aucune différence n'est apparue. Cela montre que les paramètres de recouvrement, d'homogénéité du faisceau et d'aspiration des particules permettent un nettoyage efficace et homogène de toute la surface.

\section{CONCLUSION}

Ces travaux ont montré que la longueur d'onde de $308 \mathrm{~nm}$ est bien adaptée pour nettoyer une surface d'acier inoxydable ou de $\mathrm{B}_{4} \mathrm{C}$ recouverte d'une couche de $20 \mathrm{~nm}$ d'or. L'étude théorique a permis de mettre en évidence qu'à $1,06 \mu \mathrm{m}$ (laser $\mathrm{Nd}: \mathrm{YAG}$ ), il fallait utiliser des densités d'énergie laser très supérieures à celles requises à $308 \mathrm{~nm}$. Le risque de diffusion de l'or dans le bain de fusion est également beaucoup plus important dans le cas du laser Nd :YAG. Toujours à partir de cette étude théorique simplifiée, il a été montré que les différences d'interaction laser-matière à $308 \mathrm{~nm}$ (laser XeCl) et $193 \mathrm{~nm}$ (laser ArF) étaient minimes. L'étude expérimentale montre que pour une densité d'énergie supérieure à $3 \mathrm{~J} / \mathrm{cm}^{2}$ et 8 tirs plus de $95 \%$ du dépôt d'or est enlevé (laser $\mathrm{XeCl})$. La présence d'or résiduel après traitement $(<1 \%$ pour l'acier et $5 \%$ pour $\mathrm{B}_{4} \mathrm{C}$ ) est due au redépôt et à la diffusion dans le bain de fusion, et, à la diffusion intergranulaire pour $\mathrm{B}_{4} \mathrm{C}$. Le système d'aspiration utilisé est bien adapté, les différents moyens d'analyse utilisés n'ayant pas permis de faire la différence en dosage d'or sur toutes les parties de l'échantillon balayé. 